\title{
BALLS IN QUATERNIONIC HYPERBOLIC MANIFOLDS
}

\author{
Wensheng CAO
}

\begin{abstract}
By use of the Zassenhaus neighborhood of $\operatorname{Sp}(n, 1)$, we obtain an explicit lower bound for the radius of the largest inscribed ball in quaternionic hyperbolic n-manifold $\mathscr{M}=\mathbf{H}_{\mathbf{H}}^{n} / \Gamma$. As an application, we obtain a lower bound for the volumes of quaternionic hyperbolic n-manifolds.
\end{abstract}

\section{Introduction}

Let $\mathbf{F}$ be real numbers $\mathbf{R}$, complex numbers $\mathbf{C}$ or quaternions $\mathbf{H}$, and $\mathbf{H}_{\mathrm{F}}^{n}$ the $n$-dimensional hyperbolic space over $\mathbf{F}$. Let $G$ be the linear groups which act as the isometries in $\mathbf{H}_{\mathbf{F}}^{n}$. For $\mathbf{F}=\mathbf{R}, \mathbf{C}$ and $\mathbf{H}, G$ are usually denoted by $\operatorname{PO}(n, 1)$, $\operatorname{PU}(n, 1)$ and $\operatorname{PSp}(n, 1)$, respectively. The elements of $G$ are classified into three types: elliptic, parabolic and loxodromic according to the cardinality of their fixed points and their locations [6]. The quotient $\mathbf{H}_{\mathbf{F}}^{n} / \Gamma$ of $\mathbf{H}_{\mathbf{F}}^{n}$ by a discrete subgroup $\Gamma$ of $G$ is denoted by $\mathscr{2}$. Such a quotient $\mathscr{2}$ is called a hyperbolic n-manifold $\mathscr{M}$ if $\Gamma$ contains no elements of finite order, and a hyperbolic n-orbifold $\mathcal{O}$ otherwise. A quotient $\mathscr{Q}$ is closed (or compact) when $\Gamma$ does not contain parabolic elements and cusped (or noncompact) when it does; and arithmetic when $\Gamma$ can be derived by a specific number-theoretic construction.

We can descend the volume form from $\mathbf{H}_{\mathbf{F}}^{n}$ to $\mathscr{Q}$ and integrate it over the quotient space. This defines the hyperbolic volume of 2 . The hyperbolic volume is an important topological invariant. For example, the famous Mostow-Prasad rigidity theorem implies that every geometric invariant of a finite volume hyperbolic n-manifold (or orbifold) of dimension $n \geq 3$ is a topological invariant.

Work by Jørgensen and Thurston says that the hyperbolic volumes of complete real hyperbolic 3-manifolds form a well-ordered subset of $\mathbf{R}$ of order type $\omega^{\omega}$ [25]. In the general case, the results of Wang and Gunther imply that the hyperbolic volumes of $\mathscr{2}$ form a discrete subset of $\mathbf{R}[9,26,27]$. Moreover, for each dimension $n \geq 3$ the number of the isometry classes of the quotients with the same volume is finite. These facts ensure the existence of

2000 Mathematics Subject Classification. Primary 30F40; Secondary 20H10, 57S30.

Key words and phrases. Quaternionic hyperbolic manifolds, inscribed ball, volume. Received April 22, 2015; revised November 17, 2015. 
the minimal volumes of certain classes of hyperbolic manifolds and orbifolds. The search of promising candidates for hyperbolic manifolds and orbifolds of minimal volume has long been a topic of interest. In general speaking, such works will involve several methods ranging from analytical, combinatorial to arithmetic techniques.

For a cusp quotient 2 , one often involves in finding the maximal horoball quotient embed in 2 and then approximate the volume of such a quotient. One of the most important tools is Shimizu's lemma, which gives a necessary condition for a subgroup of $\operatorname{PSL}(2, \mathbf{R})$ containing a parabolic element to be discrete. In geometric point of view, it says that the horoball of a parabolic element is precisely invariant (that is elements of the group either map the horoball to itself or to a disjoint horoball). Therefore, Shimizu's lemma may be thought of as an effective version of the Margulis constant in the case of cusps. There have been many generalizations in $\mathbf{H}_{\mathbf{R}}^{n}$ and $\mathbf{H}_{\mathbf{C}}^{n}$. Such generalizations play an important role in estimating the minimal volumes in $[14,15,16,24]$. Recently Cao and Parker [4] obtained a full version of Shimizu's lemma for any parabolic isometry of $\mathbf{H}_{\mathbf{C}}^{n}$ or $\mathbf{H}_{\mathbf{H}}^{n}$ for all $n \geq 2$.

For a compact quotient 2 , one often involves in finding a maximal embedded tubular neighborhood of the simple closed geodesic in 2 . One of the most important tools is Jørgensen's inequality, which gives a necessary condition for a non-elementary two generator subgroup of $\operatorname{PSL}(2, \mathbf{C})$ to be discrete. Shimizu's lemma is a special case of Jørgensen's inequality. Cao and Parker [3] obtained analogues of Jørgensen's inequality for non-elementary groups of isometries of quaternionic hyperbolic $n$-space generated by two elements, one of which is loxodromic. They used the quaternionic version of Jørgensen's inequalities to construct embedded collars about short, simple, closed geodesics in quaternionic hyperbolic manifolds and showed that these canonical collars are disjoint from each other.

Adeboye obtained an explicit lower bound for the volume of a real hyperbolic orbifold depending on the dimension [1]. The main tool is the spectral radius of the involved matrices. Such a technique was employed by $\mathrm{Fu}$ and Wang to treat the analogous problem in complex hyperbolic spaces [8]. Recently Adeboye and Wei reconsidered such a question using the theory of Lie group and Lie algebra [2]. Let $G$ be a semisimple Lie group without compact factor, let $i d$ be the identity of $G$, let $\rho$ be the distance function derived from a canonical metric, and let

$$
B\left(i d, R_{G}\right)=\left\{x \in G: \rho(i d, x) \leq R_{G}\right\} .
$$

Wang [26] obtained that for any discrete subgroup $\Gamma$ of $G$, there exists $f \in G$ such that

$$
B\left(i d, R_{G}\right) \cap f \Gamma f^{-1}=\{i d\} .
$$

Adeboye and Wei used the above result to produce an inscribed metric ball on the fundamental domain of $\mathrm{SU}(n, 1) / \Gamma$. And then they obtained their main result in [2] mainly by Gunther's result [9] and the Riemannian submersion 
$\Pi: \operatorname{SU}(n, 1) / \Gamma \rightarrow \mathbf{H}_{\mathbf{C}}^{n} / \Gamma$. One can refer to the last section of [2] for more current results on complex hyperbolic volume.

We mention that the mechanism of lower bound of the volume of quotient 2 in geometry is mainly attributed to the isolation of the identity in $\Gamma$ in algebra. Jørgensen's inequality and Wang's result are useful tools to describe such isolation in quantity in different categories. They all provide different types of quantitative version of the well-known result of Kazhdan-Margulis [18].

For simplicity, we focus on the case of manifolds in what follows. Let $\mathscr{F}_{n}$ be the set of all hyperbolic n-manifolds and define

$$
r_{n}=\frac{1}{2} \inf _{\Gamma \in \mathscr{F}_{n}} \sup _{o \in \mathbf{H}_{\mathbf{F}}^{n}} \inf _{f \in \Gamma} \rho(o, f(o)),
$$

where $\rho(\cdot, \cdot)$ is the hyperbolic distance in $\mathbf{H}_{\mathbf{F}}^{n}$.

It is obvious that $r_{n}$ is the largest number such that every hyperbolic n-manifold contains an inscribed ball of that radius. A positive lower bound on $r_{n}$ will provide geometric information about all hyperbolic n-manifolds, such as the lower bounds for the volumes of hyperbolic manifolds and the thick and thin decomposition of such manifolds. We mention that such a ball is contained in a canonical collar for a compact manifold and it may be contained in a cusp for a cusp manifold. For example, Parker showed that there is an embedded ball of a fixed size in any complex hyperbolic orbifold with a pure parabolic cusp [23].

It has long been known that for each dimension there is a lower bound of $r_{n}$ in real hyperbolic space. This follows from a theorem of Kazdan and Margulis [18] in their proof of Selberg's hypothesis, see also in $[26,29]$. The method of proof found by these authors consists of a compactness argument together with the existence of Zassenhaus neighborhoods.

For the real hyperbolic 3-manifolds, Waterman [28] obtained the bound $r_{3} \geq 0.04000$. Gering and Martin [10] improved this bound to $r_{3} \geq 0.05725$. By use of the generalized Jørgensen's inequality [20], Martin [21] obtained an explicit lower bound for $r_{n}$ depending only on the dimension $n$. Using the Jørgensen's inequality for discrete groups in normed algebras, Friedland and Hersonsky [7] improved this result to

$$
r_{n}>\frac{0.0025}{17^{[n / 2]}},
$$

where $[n / 2]$ is the integer part of $n / 2$.

As interest in quaternionic hyperbolic space has grown, many of real and complex hyperbolic problems have been translated into the quaternionic arena, in which the problems are almost always vastly more complicated $[3,4,5,19]$.

In this paper, we consider the lower bounds of $r_{n}$ in quaternionic hyperbolic manifolds. Let $\Gamma$ be a discrete non-elementary torsion free subgroup of $\operatorname{Sp}(n, 1)$. Motivated by the ideas of Martin, Friedland and Hersonsky [21, 7], we will obtain in Section 3 that there is an $f \in \operatorname{Sp}(n, 1)$ such that

$$
\|h\|\left\|h-I_{n+1}\right\| \geq \omega
$$


for all $h \in f \Gamma f^{-1}$, where $\omega \approx 0.385458$. This formula is crucial for our construction of inscribed ball in quaternionic hyperbolic manifolds.

Our main result is the following theorem.

THeOREM 1.1. Let $\mathscr{M}=\mathbf{H}_{\mathbf{H}}^{n} / \Gamma$ be a quaternionic hyperbolic $n$-manifold. Let $r_{n}$ be given by (1). Then

$$
r_{n}>\frac{0.0114}{17^{n+1}}
$$

We will show the following proposition in Section 4.

Proposition 1.1. The quaternionic hyperbolic volume of a geodesic ball of radius $\rho$ is

$$
\operatorname{Vol}(B(\rho))=\frac{(4 \pi)^{2 n}}{(2 n+1) !} \sinh ^{4 n}\left(\frac{\rho}{2}\right)\left[1+2 n \cosh ^{2}\left(\frac{\rho}{2}\right)\right] .
$$

As an application of Theorem 1.1, we obtain a lower bound for the volume of a quaternionic hyperbolic n-manifold. Then

COROLlary 1.1. Let $\mathscr{M}=\mathbf{H}_{\mathbf{H}}^{n} / \Gamma$ be a quaternionic hyperbolic n-manifold.

$$
\operatorname{Vol}(\mathscr{M})>\frac{(4 \pi)^{2 n}}{(2 n+1) !} \sinh ^{4 n}\left(\frac{0.0057}{17^{n+1}}\right)\left[1+2 n \cosh ^{2}\left(\frac{0.0057}{17^{n+1}}\right)\right]
$$

We would like to mention that although the above formula gives the first explicit lower bounds for the volumes of quaternionic hyperbolic manifolds that depend only on dimension, we believe that the lower bounds can be improved rapidly when we consider the each special cases with more special tool for involved categories of manifolds. To some extent, this is mainly due to the facts that the inscribed ball is just a part of the tube or the horoball involved and general method is not so effective for each special cases.

The paper is organized as follows. Section 2 contains some necessary background material for quaternionic hyperbolic geometry. In Section 3, we obtain the Zassenhaus neighborhood of $\operatorname{Sp}(n, 1)$. From this we obtain the characteristic (2) of the isolation of the identity in discrete subgroup $\Gamma$. In Section 4, we obtain the volume form of ball model of quaternionic hyperbolic n-space. From this we deduce the volume formula of hyperbolic geodesic ball of radius $\rho$. Section 5 contains the proof of Theorem 1.1. Section 6 contains an analogue result in complex hyperbolic geometry.

\section{Preliminaries}

In this section, we give some necessary background materials of quaternionic hyperbolic geometry. More details can be found in $[5,6,11,19]$. 
Let $\mathbf{H}^{n, 1}$ be the vector space of dimension $n+1$ over $\mathbf{H}$ with the unitary structure defined by the Hermitian form

$$
\langle\mathbf{z}, \mathbf{w}\rangle=\mathbf{w}^{*} J \mathbf{z}=\overline{w_{1}} z_{1}+\cdots+\overline{w_{n}} z_{n}-\overline{w_{n+1}} z_{n+1},
$$

where $\mathbf{z}$ and $\mathbf{w}$ are the column vectors in $V$ with entries $\left(z_{1}, \ldots, z_{n+1}\right)$ and $\left(w_{1}, \ldots, w_{n+1}\right)$ respectively, .* denotes the conjugate transpose and $J$ is the Hermitian matrix

$$
J=\left(\begin{array}{cc}
I_{n} & 0 \\
0 & -1
\end{array}\right)
$$

We define a unitary transformation $g$ to be an automorphism of $\mathbf{H}^{n, 1}$, that is, a linear bijection such that $\langle g(\mathbf{z}), g(\mathbf{w})\rangle=\langle\mathbf{z}, \mathbf{w}\rangle$ for all $\mathbf{z}$ and $\mathbf{w}$ in $V$. We denote the group of all unitary transformations by $\operatorname{Sp}(n, 1)$.

Following Section 2 of [6], let

$$
\begin{aligned}
& V_{0}=\{\mathbf{z} \in V-\{0\}:\langle\mathbf{z}, \mathbf{z}\rangle=0\}, \\
& V_{-}=\{\mathbf{z} \in V:\langle\mathbf{z}, \mathbf{z}\rangle\langle 0\} .
\end{aligned}
$$

It is obvious that $V_{0}$ and $V_{-}$are invariant under $\operatorname{Sp}(n, 1)$. We define $V^{s}$ to be $V^{s}=V_{-} \cup V_{0}$. Let $P: V^{s} \rightarrow P\left(V^{s}\right) \subset \mathbf{H}^{n}$ be the projection map defined by

$$
P\left(z_{1}, \ldots, z_{n}, z_{n+1}\right)^{t}=\left(z_{1} z_{n+1}^{-1}, \ldots, z_{n} z_{n+1}^{-1}\right)^{t},
$$

where ${ }^{t}$ denotes the transpose.

We define $\mathbf{H}_{\mathbf{H}}^{n}=P\left(V_{-}\right)$and $\partial \mathbf{H}_{\mathbf{H}}^{n}=P\left(V_{0}\right)$. The Bergman metric on $\mathbf{H}_{\mathbf{H}}^{n}$ is given by the distance formula

$$
\cosh ^{2} \frac{\rho(z, w)}{2}=\frac{\langle\mathbf{z}, \mathbf{w}\rangle\langle\mathbf{w}, \mathbf{z}\rangle}{\langle\mathbf{z}, \mathbf{z}\rangle\langle\mathbf{w}, \mathbf{w}\rangle}, \quad \text { where } \mathbf{z} \in P^{-1}(z), \mathbf{w} \in P^{-1}(w) .
$$

Alternatively, the Bergman metric [22] is given by

$$
d s^{2}=\frac{-4}{\langle\mathbf{z}, \mathbf{z}\rangle} \operatorname{det}\left(\begin{array}{cc}
\langle\mathbf{z}, \mathbf{z}\rangle & \langle d \mathbf{z}, \mathbf{z}\rangle \\
\langle\mathbf{z}, d \mathbf{z}\rangle & \langle d \mathbf{z}, d \mathbf{z}\rangle
\end{array}\right) .
$$

The holomorphic isometry group of $\mathbf{H}_{\mathbf{H}}^{n}$ with respect to the Bergman metric is the projective unitary group $\operatorname{PSp}(n, 1)=\operatorname{Sp}(n, 1) / \pm I_{n+1}$ and acts on $P\left(\mathbf{H}^{n, 1}\right)$ by matrix multiplication.

If $g \in \mathrm{Sp}(n, 1)$, by definition, $g$ preserves the Hermitian form. Hence

$$
\mathbf{w}^{*} J \mathbf{z}=\langle\mathbf{z}, \mathbf{w}\rangle=\langle g \mathbf{z}, g \mathbf{w}\rangle=\mathbf{w}^{*} g^{*} J g \mathbf{z}
$$

for all $\mathbf{z}$ and $\mathbf{w}$ in $V$. Letting $\mathbf{z}$ and $\mathbf{w}$ vary over a basis for $V$ we see that $J=g^{*} J g$. From this we find $g^{-1}=J g^{*} J$. That is:

$$
g^{-1}=\left(\begin{array}{cc}
A^{*} & -\beta^{*} \\
-\alpha^{*} & \frac{1}{a_{n+1, n+1}}
\end{array}\right) \text { for } g=\left(a_{i j}\right)_{i, j=1, \ldots, n+1}=\left(\begin{array}{cc}
A & \alpha \\
\beta & a_{n+1, n+1}
\end{array}\right) .
$$


Using the identities $g g^{-1}=g^{-1} g=I$ we obtain:

$$
\begin{aligned}
& A A^{*}-\alpha \alpha^{*}=I_{n}, \quad-A \beta^{*}+\alpha \overline{a_{n+1, n+1}}=0, \quad-|\beta|^{2}+\left|a_{n+1, n+1}\right|^{2}=1 ; \\
& A^{*} A-\beta^{*} \beta=I_{n}, \quad A^{*} \alpha-\beta^{*} a_{n+1, n+1}=0, \quad-|\alpha|^{2}+\left|a_{n+1, n+1}\right|^{2}=1 .
\end{aligned}
$$

Following Chen and Greenberg [6], we say that a non-trivial element $g$ of $\operatorname{Sp}(n, 1)$ is:

(i) elliptic if it has a fixed point in $\mathbf{H}_{\mathbf{H}}^{n}$;

(ii) parabolic if it has exactly one fixed point which lies in $\partial \mathbf{H}_{\mathbf{H}}^{n}$;

(iii) loxodromic if it has exactly two fixed points which lie in $\partial \mathbf{H}_{\mathbf{H}}^{n}$.

If $g \in \operatorname{Sp}(n, 1)$ has fixed point $q_{0}=(0, \ldots, 0)^{t} \in \mathbf{H}_{\mathbf{H}}^{n}$, then $g$ has the form

$$
g=\operatorname{diag}(A, a),
$$

where $A \in U(n ; \mathbf{H})$ and $a \in U(1 ; \mathbf{H})$. We denote such an isotropy group of $q_{0}$ by $G_{0}$. We mention that $G_{0}$ contains elliptic elements of the form $g=a I_{n+1}$, $a \in U(1 ; \mathbf{H})$. Such a type of elliptic elements is exclusive in $\operatorname{Sp}(n, 1)$. For $a \notin \mathbf{R}$, the set of fixed points of such an element is a totally geodesic submanfold which is equivalent to $\mathbf{H}_{\mathbf{C}}^{n}$ [6].

\section{The Zassenhaus neighborhood of $\operatorname{Sp}(n, 1)$}

For an element $A$ of $M_{n+1}(\mathbf{H})$, the algebra of $(n+1) \times(n+1)$ quaternion valued matrices, let $\sigma(A)$ denote the right spectra of $A$, i.e., the set of all right eigenvalues of $A$. If $\lambda \in \sigma(A)$ then there exist nonzero vector $v \in \mathbf{H}^{n+1}$ such that $A v=v \lambda$. Thus $A v u^{-1}=v u^{-1} u \lambda u^{-1}$ for any $0 \neq u \in \mathbf{H}$, i.e., $u \lambda u^{-1} \in \sigma(A)$. Right eigenvalues are conjugacy invariants for matrices $A \in M_{n+1}(\mathbf{H})$ [30]. The number

$$
r_{\sigma}(A)=\max _{\lambda \in \sigma(A)}|\lambda|
$$

is defined to be the right spectral radius of $A$. We denote its spectral norm as

$$
\|A\|=\sqrt{r_{\sigma}\left(A^{*} A\right)} .
$$

As in the complex case [17], we can show that the above norm is a matrix norm induced by the vector norm on $\mathbf{H}^{n+1}$. That is

$$
\|A\|=\max \left\{|A v|: v \in \mathbf{H}^{n+1} \text { and }|v|=1\right\} .
$$

This norm has the following properties:

$$
r_{\sigma}(A) \leq\|A\|, \quad\left\|A^{*}\right\|=\|A\|=\|U A V\|, \quad \forall U, V \in U(n+1, \mathbf{H}) .
$$

It is obvious that

$$
\|g\|=1, \quad \forall g \in G_{0} .
$$

We mention that this norm isn't a conjugacy invariant. 
Proposition 3.1. Let $g \in \mathrm{Sp}(n, 1)$. Then

$$
\left\|g^{-1}-I_{n+1}\right\|=\left\|g-I_{n+1}\right\|, \quad\left\|g^{-1}\right\|=\|g\| \leq 1+\left\|g-I_{n+1}\right\| .
$$

Proof. Since $g^{-1}=J g^{*} J$, we have

$$
\left\|g^{-1}-I_{n+1}\right\|=\left\|J g^{*} J-I_{n+1}\right\|=\left\|J\left(g-I_{n+1}\right)^{*} J\right\|=\left\|g-I_{n+1}\right\|
$$

and

$$
\left\|g^{-1}\right\|=\left\|J g^{*} J\right\|=\|g\|=\left\|I_{n+1}+g-I_{n+1}\right\| \leq 1+\left\|g-I_{n+1}\right\| .
$$

Set

$$
B_{0}\left(I_{n+1}, r\right)=\left\{X \in M_{n+1}(\mathbf{H}):\left\|X-I_{n+1}\right\|<r\right\}
$$

and

$$
\tilde{B}_{0}\left(I_{n+1}, r\right)=\left\{X \in M_{n+1}(\mathbf{H}):\|X\|\left\|X-I_{n+1}\right\|<r\right\} .
$$

Denote by $\tau$ the positive solution of

$$
2 \tau(1+\tau)^{2}=1, \quad \tau \approx 0.297156
$$

We obtain the Zassenhaus neighborhood of $\operatorname{Sp}(n, 1)$ as follows.

THEOREM 3.1. Let $\Gamma$ be discrete subgroup of $\operatorname{Sp}(n, 1)$. For any $0<r<\tau$, the group generated by the elements $\Gamma \cap B_{0}\left(I_{n+1}, r\right)$ is nilpotent. That is, there exists an $m$ so that for any sequence:

$$
f_{i} \in B_{0}\left(I_{n+1}, r\right), \quad i=0,1, \ldots, \quad h_{i}=\left[f_{i}, h_{i-1}\right], \quad i=1,2, \ldots, h_{0}=f_{0},
$$

$h_{m}=I_{n+1}$. In particular, if the subgroup $\langle f, g\rangle$ generated by $f, g \in \operatorname{Sp}(n, 1)$ is discrete group and

$$
\left\|f-I_{n+1}\right\|<\tau, \quad\left\|g-I_{n+1}\right\|<\tau,
$$

then $\langle f, g\rangle$ is a nilpotent group.

Proof. Let $c=r / \tau<1$. We claim that

$$
\left\|h_{i}-I_{n+1}\right\|<c^{i+1} \tau, \quad i=0,1, \ldots
$$

We proof this claim by induction. As $h_{0}=f_{0} \in B_{0}\left(I_{n+1}, r\right)$. (13) holds trivially. Assume that (13) holds for $i=k$. Note that $c<1$ and $2 \tau(1+\tau)^{2}=1$. By Proposition 3.1, we have

$$
\begin{aligned}
\left\|h_{k+1}-I_{n+1}\right\| & =\left\|\left(f_{k+1} h_{k}-h_{k} f_{k+1}\right) f_{k+1}^{-1} h_{k}^{-1}\right\| \\
& =\left\|\left[\left(f_{k+1}-I_{n+1}\right)\left(h_{k}-I_{n+1}\right)-\left(h_{k}-I_{n+1}\right)\left(f_{k+1}-I_{n+1}\right)\right] f_{k+1}^{-1} h_{k}^{-1}\right\| \\
& \leq 2\left\|f_{k+1}-I_{n+1}\right\|\left\|h_{k}-I_{n+1}\right\|\left\|f_{k+1}^{-1}\right\|\left\|h_{k}^{-1}\right\| \\
& \leq 2\left\|f_{k+1}-I_{n+1}\right\|\left\|h_{k}-I_{n+1}\right\|\left(1+\left\|f_{k+1}-I_{n+1}\right\|\right)\left(1+\left\|h_{k}-I_{n+1}\right\|\right)
\end{aligned}
$$




$$
\begin{aligned}
& <2 c \tau(1+c \tau)\left(1+c^{k+1} \tau\right) c^{k+1} \tau \\
& <c^{k+2} \tau\left[2 \tau(1+\tau)^{2}\right] \\
& =c^{k+2} \tau .
\end{aligned}
$$

Since $\Gamma$ is discrete, there exists a $\varepsilon$ such that $\Gamma \cap B_{0}\left(I_{n+1}, \varepsilon\right)=I_{n+1}$. Let $m$ be the smallest positive integer so that $c^{m+1} \tau<\varepsilon$. Then (13) implies that $h_{m} \in$ $B_{0}\left(I_{n+1}, \varepsilon\right)$. Hence $h_{m}=I_{n+1}$ and this implies that the group generated by the elements $\Gamma \cap B_{0}\left(I_{n+1}, r\right)$ is nilpotent.

Suppose that (12) holds. Consider the sequence (11) with $f_{i}=f, i=$ $0,1, \ldots$ We can get $h_{m}=I_{n+1}$ and this implies that $\langle f, g\rangle$ is a nilpotent group.

Denote by $\omega$ the positive solution of

$$
2 \omega\left(2 \omega^{2}+1\right)=1, \quad \omega \approx 0.385458 .
$$

Since the norm of all right eigenvalue of $g \in \operatorname{Sp}(n, 1)$ is bagger that 1 , we have that $\|g\| \geq 1$. Hence $\left\|g-I_{n+1}\right\| \leq\|g\|\left\|g-I_{n+1}\right\|$, we get

$$
\tilde{B}_{0}\left(I_{n+1}, r\right) \subset B_{0}\left(I_{n+1}, r\right) \text {. }
$$

We need the following theorem for our purpose.

THEOREM 3.2. Let $\Gamma$ be discrete subgroup of $\mathrm{Sp}(n, 1)$. For any $0<r<\omega$, the group generated by the elements $\Gamma \cap \widetilde{B}_{0}\left(I_{n+1}, r\right)$ is nilpotent.

Proof. Consider the following sequence

$$
f_{i} \in \tilde{B}_{0}\left(I_{n+1}, r\right), \quad i=0,1, \ldots, \quad h_{i}=\left[f_{i}, h_{i-1}\right], \quad i=1,2, \ldots, h_{0}=f_{0} .
$$

Let $c=r / \omega<1$. We claim that

$$
\left\|h_{i}\right\|\left\|h_{i}-I_{n+1}\right\|<c^{i+1} \omega, \quad i=0,1, \ldots
$$

As $h_{0}=f_{0} \in \tilde{B}_{0}\left(I_{n+1}, r\right)$. (15) holds trivially. Assume that (15) holds for $i=k$. Note that $f_{k+1} \in \tilde{B}_{0}\left(I_{n+1}, r\right)$. As in the proof of Theorem 3.1, we get

$$
\begin{aligned}
\left\|h_{k+1}-I_{n+1}\right\| & \leq 2\left\|f_{k+1}-I_{n+1}\right\|\left\|h_{k}-I_{n+1}\right\|\left\|f_{k+1}^{-1}\right\|\left\|h_{k}^{-1}\right\| \\
& =2\left\|f_{k+1}\right\|\left\|f_{k+1}-I_{n+1}\right\|\left\|h_{k}\right\|\left\|h_{k}-I_{n+1}\right\| \\
& <2 c^{k+2} \omega^{2} .
\end{aligned}
$$

Therefore

$$
\begin{aligned}
\left\|h_{k+1}\right\|\left\|h_{k+1}-I_{n+1}\right\| & \leq\left(1+\left\|h_{k+1}-I_{n+1}\right\|\right)\left\|h_{k+1}-I_{n+1}\right\| \\
& <\left(1+2 c^{k+2} \omega^{2}\right) 2 c^{k+2} \omega^{2} \\
& <\left(1+2 \omega^{2}\right) 2 c^{k+2} \omega^{2}=c^{k+2} \omega .
\end{aligned}
$$


Since $\Gamma$ is discrete, there exists a $\varepsilon$ such that $\Gamma \cap B_{0}\left(I_{n+1}, \varepsilon\right)=I_{n+1}$. Let $m$ be the smallest positive integer so that $c^{m+1} \omega<\varepsilon$. Then (15) implies that $h_{m} \in$ $\tilde{B}_{0}\left(I_{n+1}, \varepsilon\right)$. Hence $h_{m}=I_{n+1}$ and this implies that the group generated by the elements $\Gamma \cap \tilde{B}_{0}\left(I_{n+1}, r\right)$ is nilpotent.

By Theorem 3.2, we have the following corollary, which is the crucial result in our construction of inscribed geodesic ball.

COROLlaRY 3.1. Let $\Gamma$ be a discrete non-elementary torsion free subgroup of $\operatorname{Sp}(n, 1)$. Then there is an $f \in \operatorname{Sp}(n, 1)$ such that

$$
\|h\|\left\|h-I_{n+1}\right\| \geq \omega
$$

for all $h \in f \Gamma f^{-1}$.

\section{The volume form of ball model}

In this section, we will obtain the volume form of ball model in quaternionic hyperbolic n-space. Using this volume form, we obtain a volume formula of geodesic balls. The volume form of Siegel domain in quaternionic hyperbolic $\mathrm{n}$-space can be found in [19].

Proposition 4.1. In the ball model of $\mathbf{H}_{\mathbf{H}}^{n}$, let $r^{2}=\sum_{i=1}^{n}\left|z_{i}\right|^{2}$. Then the volume form is given by

$$
d \mathrm{Vol}=\frac{4^{2 n}}{\left(1-r^{2}\right)^{2(n+1)}} d v o l
$$

where $d v o l=r^{4 n-1} d r d \sigma$ and $\sigma$ is the Euclidean volume of the unit $(4 n-1)$ real sphere.

Proof. Let $z=\left(z_{1}, z_{2}, \ldots, z_{n}\right) \in \mathbf{H}_{\mathbf{H}}^{n}$ and $r=|z|$. Substituting in (5), we get

$$
d s^{2}=\frac{4\left(1-r^{2}\right)\left(\left|d z_{1}\right|^{2}+\cdots\left|d z_{n}\right|^{2}\right)+4\left|\overline{z_{1}} d z_{1}+\cdots+\overline{z_{n}} d z_{n}\right|^{2}}{\left(r^{2}-1\right)^{2}}
$$

Let $z_{i}=x_{i}+y_{i} \mathbf{i}+v_{i} \mathbf{j}+w_{i} \mathbf{k}$, where $x_{i}, y_{i}, v_{i}, w_{i} \in \mathbf{R}$ and

$$
\mathbf{i}^{2}=\mathbf{j}^{2}=\mathbf{k}^{2}=\mathbf{i j k}=-1 \text {. }
$$

Note that $\left|d z_{1}\right|^{2}+\cdots\left|d z_{n}\right|^{2}=\sum_{i=1}^{n}\left(d x_{i}^{2}+d y_{i}^{2}+d v_{i}^{2}+d w_{i}^{2}\right)$ and

$$
\begin{aligned}
\left|\sum_{i=1}^{n} \overline{z_{i}} d z_{i}\right|^{2}= & {\left[\sum_{i=1}^{n}\left(x_{i} d x_{i}+y_{i} d y_{i}+v_{i} d v_{i}+w_{i} d w_{i}\right)\right]^{2} } \\
& +\left[\sum_{i=1}^{n}\left(x_{i} d y_{i}-y_{i} d x_{i}+w_{i} d v_{i}-v_{i} d w_{i}\right)\right]^{2}
\end{aligned}
$$




$$
\begin{aligned}
& +\left[\sum_{i=1}^{n}\left(x_{i} d v_{i}-v_{i} d x_{i}+y_{i} d w_{i}-w_{i} d y_{i}\right)\right]^{2} \\
& +\left[\sum_{i=1}^{n}\left(x_{i} d w_{i}-w_{i} d x_{i}+v_{i} d y_{i}-y_{i} d v_{i}\right)\right]^{2} .
\end{aligned}
$$

Then we can rewrite the metric (16) as

$$
\begin{aligned}
d s^{2}= & \left(d x_{1}, d y_{1}, d v_{1}, d w_{1}, \ldots, d x_{n}, d y_{n}, d v_{n}, d w_{n}\right) g_{\mathbf{R}} \\
& \left(d x_{1}, d y_{1}, d v_{1}, d w_{1}, \ldots, d x_{n}, d y_{n}, d v_{n}, d w_{n}\right)^{T} .
\end{aligned}
$$

At the point $P=(0, \ldots, r) \in \mathbf{H}_{\mathbf{H}}^{n}$, we have

$$
\left.g_{\mathbf{R}}\right|_{P}=\frac{4}{\left(1-r^{2}\right)^{2}} \operatorname{diag}\left(1-r^{2}, \ldots, 1-r^{2}, 1,1,1,1\right) .
$$

By computation, we can get

Hence the volume form is

$$
\operatorname{det}_{\mathbf{R}}=\frac{4^{4 n}}{\left(1-r^{2}\right)^{4(n+1)}}
$$

$$
d \mathrm{Vol}=\sqrt{g_{\mathbf{R}}} d v o l=\frac{4^{2 n}}{\left(1-r^{2}\right)^{2(n+1)}} d v o l .
$$

With this volume form, we are ready to prove Proposition 1.1.

The proof of Proposition 1.1. Since the Euclidean distance $r$ from 0 is related to the hyperbolic distance $\rho$ by

$$
r=\tanh \left(\frac{\rho}{2}\right)
$$

By Proposition 4.1, we have

$$
\begin{aligned}
\operatorname{Vol}(B(\rho)) & =\int_{2 \tanh ^{-1}(r) \leq \rho} \frac{4^{2 n}}{\left(1-r^{2}\right)^{2(n+1)}} r^{4 n-1} d r d \sigma \\
& =4^{2 n} \sigma_{4 n-1} \int_{0}^{\tanh (\rho / 2)} \frac{r^{4 n-1}}{\left(1-r^{2}\right)^{2(n+1)}} d r .
\end{aligned}
$$

Let $r=\tanh \left(\frac{\delta}{2}\right)=\frac{e^{\delta}-1}{e^{\delta}+1}$. Note that $\sigma_{4 n-1}=\frac{2 \pi^{2 n}}{(2 n-1) !}$. We have

$$
\begin{aligned}
\operatorname{Vol}(B(\rho)) & =\frac{\sigma_{4 n-1}}{2} 4^{2 n} \int_{0}^{\rho} \cosh ^{3}\left(\frac{\delta}{2}\right) \sinh ^{4 n-1}\left(\frac{\delta}{2}\right) d \delta \\
& =4^{2 n} \sigma_{4 n-1} \int_{0}^{\rho}\left[\sinh ^{4 n+1}\left(\frac{\delta}{2}\right)+\sinh ^{4 n-1}\left(\frac{\delta}{2}\right)\right] d \sinh \left(\frac{\delta}{2}\right)
\end{aligned}
$$




$$
\begin{aligned}
& =\left.4^{2 n} \sigma_{4 n-1}\left[\frac{\sinh ^{4 n+2}\left(\frac{\delta}{2}\right)}{4 n+2}+\frac{\sinh ^{4 n}\left(\frac{\delta}{2}\right)}{4 n}\right]\right|_{\delta=0} ^{\delta=\rho} \\
& =4^{2 n} \sigma_{4 n-1} \sinh ^{4 n}\left(\frac{\rho}{2}\right)\left[\frac{\sinh ^{2}\left(\frac{\rho}{2}\right)}{4 n+2}+\frac{1}{4 n}\right] \\
& =\frac{1}{(2 n+1) !}(4 \pi)^{2 n} \sinh ^{4 n}\left(\frac{\rho}{2}\right)\left[1+2 n \cosh ^{2}\left(\frac{\rho}{2}\right)\right] .
\end{aligned}
$$

Remark 4.1. Recently the author came across the fact that Gray has obtained the volume formula of quaternionic hyperbolic geodesic ball in $[12$, page 341].

Similarly we can work in complex hyperbolic space and obtain the following proposition. One can also find such a result in [13, Lemma 6.18 and Corollary A.3].

Proposition 4.2. In the ball model of $\mathbf{H}_{\mathbf{H}}^{n}$, let $r^{2}=\sum_{i=1}^{n}\left|z_{i}\right|^{2}$. Then the volume form is given by

$$
d \mathrm{Vol}=\frac{4^{n}}{\left(1-r^{2}\right)^{n+1}} d v o l,
$$

where $d v o l=r^{2 n-1} d r d \sigma$ and $\sigma$ is the Euclidean volume of the unit $(2 n-1)$ real sphere. The complex hyperbolic volume of a geodesic ball of radius $\rho$ is

$$
\operatorname{Vol}(B(\rho))=\frac{1}{n !}(4 \pi)^{n} \sinh ^{2 n}\left(\frac{\rho}{2}\right) .
$$

\section{The proof of main theorem}

In order to prove Theorem 1.1, we need the following three lemmas.

Lemma 5.1. Let $g \in \operatorname{Sp}(n, 1)$. Then

$$
\operatorname{dist}\left(g, G_{0}\right)=\inf \left\{\|g-U\|, U \in G_{0}\right\} \leq e^{t}\left(e^{t}-1\right),
$$

where $t=\frac{\rho(0, g(0))}{2}$.

Proof. Let $g$ be given by (6). Then

$$
g(0)=\alpha a_{n+1, n+1}^{-1}, \quad \cosh ^{2} \frac{\rho(0, g(0))}{2}=\left|a_{n+1, n+1}\right|^{2} .
$$


By (8), we may assume without loss of generality that $g(0)=(0, \ldots, 0, \tanh t)$. Let

$$
h=\left(\begin{array}{ccc}
I_{n-1} & & \\
& \cosh t & \sinh t \\
& \sinh t & \cosh t
\end{array}\right) \in \operatorname{Sp}(n, 1) .
$$

Then $h(0)=g(0)$ and $h^{-1} g \in G_{0}$. Note that $\|h\|=e^{t}$ and $\left\|h^{-1}-I_{n+1}\right\|=e^{t}-1$. Hence we have $\|g\|=\left\|h h^{-1} g\right\| \leq\|h\|\left\|h^{-1} g\right\| \leq\|h\|$ and

$$
\operatorname{dist}\left(g, G_{0}\right) \leq\left\|g-h^{-1} g\right\| \leq\left\|h^{-1}-I_{n+1}\right\|\|g\| \leq\left\|h^{-1}-I_{n+1}\right\|\|h\|=e^{t}\left(e^{t}-1\right) .
$$

Lemma 5.2. Let $g \in \operatorname{Sp}(n, 1)$ and $h \in G_{0}$. Then for each $j>1$,

where $t=\frac{\rho(0, g(0))}{2}$.

$$
\left\|g^{j}-h^{j}\right\| \leq \frac{e^{t j}-1}{e^{t}-1}\|g-h\| .
$$

Proof. We recall the expansion

$$
g^{j}-h^{j}=(g-h) g^{j-1}+h(g-h) g^{j-2}+\cdots+h^{j-2}(g-h) g+h^{j-1}(g-h) .
$$

Note that $\|g\| \leq e^{t}$. Hence we have

$$
\begin{aligned}
\left\|g^{j}-h^{j}\right\| & \leq\|g-h\|\left(\left\|g^{j-1}\right\|+\|h\|\left\|g^{j-2}\right\|+\cdots+\left\|h^{j-1}\right\|\right) \\
& =\|g-h\|\left(\left\|g^{j-1}\right\|+\left\|g^{j-2}\right\|+\cdots+1\right) \\
& \leq \frac{e^{t j}-1}{e^{t}-1}\|g-h\| .
\end{aligned}
$$

Lemma 5.3. Let $g \in G_{0}$. Then for each $Q>1$ there is $h \in G_{0}$ such that for some $1 \leq q \leq Q^{n+1}, h^{q}=I_{n+1}$ and

$$
\|g-h\| \leq \frac{2 \pi}{q Q}
$$

Proof. Without loss of generality, we assume that $g=\operatorname{diag}\left(e^{\mathbf{i} \theta_{1}}, \ldots, e^{\mathbf{i} \theta_{n+1}}\right)$, $\theta_{i} \in[0,2 \pi]$. Use the pigeon-hole principle, for any $Q$, there exists some $1 \leq q \leq$ $Q^{n+1}$ and $p_{i}$ such that

$$
\left|\theta_{i}-\frac{2 \pi p_{i}}{q}\right| \leq \frac{2 \pi}{q Q} .
$$

Let $h=\operatorname{diag}\left(e^{\mathbf{i}\left(2 \pi p_{1} / q\right)}, \ldots, e^{\mathbf{i}\left(2 \pi p_{n+1} / q\right)}\right)$. Then $h^{q}=I_{n+1}$ and

$$
\|g-h\|=\max _{i}\left|e^{\mathbf{i} \theta_{1}}-e^{\mathbf{i}\left(2 \pi p_{1} / q\right)}\right|=2 \max _{i}\left|\sin \frac{\theta_{i}-\frac{2 \pi p_{1}}{q}}{2}\right| \leq \frac{2 \pi}{q Q} .
$$


THEOREM 5.1. Let $\Gamma \subset \operatorname{Sp}(n, 1)$ be discrete torsion free non-elementary group. Then there exists $o \in \mathbf{H}_{\mathbf{H}}^{n}$ such that for any $g \in \Gamma$,

$$
\rho(o, g(o)) \geq \delta
$$

where $\delta=\frac{0.0228}{17^{n+1}}$.

Proof. By Corollary 3.1, we know that there exists an $f \in S p(n, 1)$ such that for any $I_{n+1} \neq h \in f \Gamma f^{-1}$,

$$
\|h\|\left\|h-I_{n+1}\right\| \geq \omega>0.38545
$$

We show that this theorem holds with $o=f^{-1}\left(q_{0}\right)$. Assume to the contrary that (18) does not hold for some $\hat{g} \in \Gamma$. Set $g=f \hat{g} f^{-1}$ and $t=\frac{\rho(0, g(0))}{2}$. Then
$e^{t}<e^{\delta / 2}$. By Lemma 5.1, there exists $h_{0} \in G_{0}$ such that

$$
\left\|g-h_{0}\right\| \leq e^{t}\left(e^{t}-1\right) .
$$
By Lemma 5.3, there exists $h_{1} \in G_{0}$ with $h_{1}^{q}=I_{n+1}$ such that $\left\|h_{1}-h_{0}\right\| \leq \frac{2 \pi}{q Q}$
and

$$
\begin{aligned}
\left\|h_{0}^{q}-I_{n+1}\right\|=\left\|h_{0}^{q}-h_{1}^{q}\right\|= & \|\left(h_{0}-h_{1}\right) h_{0}^{q-1}+h_{1}\left(h_{0}-h_{1}\right) h_{0}^{q-2} \\
& +\cdots+h_{1}^{q-2}\left(h_{0}-h_{1}\right) h_{0}+h_{1}^{q-1}\left(h_{0}-h_{1}\right) \| \leq \frac{2 \pi}{Q} .
\end{aligned}
$$

By Lemma 5.2, we have

$$
\begin{gathered}
\left\|g^{q}-h_{0}^{q}\right\| \leq e^{t}\left(e^{t q}-1\right), \\
\left\|g^{q}\right\|=\left\|g^{q}-h_{0}^{q}+h_{0}^{q}\right\| \leq e^{t}\left(e^{t q}-1\right)+1
\end{gathered}
$$

and

$$
\left\|g^{q}-I_{n+1}\right\|=\left\|g^{q}-h_{0}^{q}+h_{0}^{q}-I_{n+1}\right\| \leq e^{t}\left(e^{t q}-1\right)+\frac{2 \pi}{Q} .
$$

Noting that $q \leq Q^{n+1}$ and $e^{t}<e^{\delta / 2}$, we have

$$
\begin{aligned}
\left\|g^{q}\right\|\left\|g^{q}-I_{n+1}\right\| & \leq\left[e^{t}\left(e^{t q}-1\right)+1\right]\left[e^{t}\left(e^{t q}-1\right)+\frac{2 \pi}{Q}\right] \\
& \leq\left[e^{\delta / 2}\left(e^{\delta Q^{n+1} / 2}-1\right)+1\right]\left[e^{\delta / 2}\left(e^{\delta Q^{n+1} / 2}-1\right)+\frac{2 \pi}{Q}\right] .
\end{aligned}
$$

Let $f(x)=(x+1)\left(x+\frac{2 \pi}{Q}\right)$. Then $f(x)$ is increasing in the interview $[0, \infty)$.

Let $u$ be the positive solution of the equation

$$
(x+1)(x+2 \pi / 17)=\omega .
$$


Then

$$
u=\frac{1}{2}\left[\sqrt{\left(1+\frac{2 \pi}{17}\right)^{2}+4\left(\omega-\frac{2 \pi}{17}\right)}-1-\frac{2 \pi}{17}\right]>0.011483
$$

Using the assumption that $Q=17, \delta=\frac{0.0228}{17^{n+1}}$ and $n \geq 1$, we know that

$$
e^{0.0114 / 17^{2}}\left(e^{0.0114}-1\right)<0.01147<u \text {. }
$$

This implies that

$$
\begin{aligned}
\left\|g^{q}\right\|\left\|g^{q}-I_{n+1}\right\| & <\left[e^{0.0114 / 17^{2}}\left(e^{0.0114}-1\right)+1\right]\left[e^{0.0114 / 17^{2}}\left(e^{0.0114}-1\right)+\frac{2 \pi}{17}\right] \\
& <0.385434<\omega .
\end{aligned}
$$

The above inequality contradicts (19).

By Theorem 5.1, we have the following corollary.

COROllary 5.1. Let $\mathscr{M}=\mathbf{H}_{\mathbf{H}}^{n} / \Gamma$ be a quaternionic hyperbolic n-manifold. Then any fundamental domain corresponding to $M$ contains a hyperbolic ball of radius

$$
r=\frac{0.0114}{17^{n+1}}
$$

Since Theorem 1.1 is just the restatement of Corollary 5.1, the proof of Theorem 1.1 is complete.

\section{An analogue result in complex hyperbolic geometry} section.

We will obtain an analogue result in complex hyperbolic geometry in this

Working on the complex hyperbolic geometry, one has the privilege to improve $1 \leq q \leq Q^{n+1}$ to $1 \leq q \leq Q^{n}$ in Lemma 5.3 because of $\mathrm{PU}(n, 1)=$ $\mathrm{U}(n, 1) / U(1)[6,11]$. Thus we can reformulate (20) in complex hyperbolic geometry as

$$
\begin{aligned}
\left\|g^{q}\right\|\left\|g^{q}-I_{n+1}\right\| & \leq\left[e^{t}\left(e^{t q}-1\right)+1\right]\left[e^{t}\left(e^{t q}-1\right)+\frac{2 \pi}{Q}\right] \\
& \leq\left[e^{\delta / 2}\left(e^{\delta Q^{n} / 2}-1\right)+1\right]\left[e^{\delta / 2}\left(e^{\delta Q^{n} / 2}-1\right)+\frac{2 \pi}{Q}\right] .
\end{aligned}
$$

Note that

$$
e^{0.0114 / 17}\left(e^{0.0114}-1\right)<0.011473<u .
$$

As in Section 5, we obtain the following lemma. 
LEMMA 6.1. Let $\Gamma \subset \mathrm{PU}(n, 1)$ be discrete torsion free non-elementary group. Then there exists $o \in \mathbf{H}_{\mathbf{C}}^{n}$ such that for any $g \in \Gamma$,

$$
\rho(o, g(o)) \geq \delta
$$

where

$$
\delta=\frac{0.0228}{17^{n}}
$$

Similarly as in quaternionic hyperbolic geometry, we obtain the following theorem.

TheORem 6.1. Let $\mathscr{M}=\mathbf{H}_{\mathbf{C}}^{n} / \Gamma$ be a complex hyperbolic $n$-manifold and $r_{n}$ be given by (1). Then

$$
r_{n}>\frac{0.0114}{17^{n}}
$$

and

$$
\operatorname{Vol}(\mathscr{M})>\frac{(4 \pi)^{n}}{n !} \sinh ^{2 n}\left(\frac{0.0057}{17^{n}}\right) .
$$

Acknowledgements. This work was supported by NSF of China (10801107), NSF of Guangdong Province (2015A030313644, 2016A030313002) and Foundation for High-level Talents in Higher Education of Guangdong Province. The authors would like to thank the referee for his or her useful suggestions.

\section{REFERENCES}

[1] I. Adeboye, Lower bounds for the volume of hyperbolic n-orbifolds, Pacific J. Math. 79 (2008), 1-19.

[2] I. Adeboye and G. F. Wei, On volumes of complex hyperbolic orbifolds, Mich. Math. J. 63 (2014), 355-369.

[ 3 ] W. S. CAO AND J. R. PARKer, Jørgensen's inequality and collars in $n$-dimensional quaternionic hyperbolic space, Quart. J. Math. 62 (2011), 523-543.

[4] W. S. Cao and J. R. Parker, Shimizu's lemma for quaternionic hyperbolic space, http:// maths.dur.ac.uk/dma0jrp/img/Quat-Shimizu.pdf.

[5] W. S. CAO AND H. O. TAN, Jørgensen's inequality for quaternionic hyperbolic space with elliptic elements, Bull. Austral. Math. Soc. 81 (2010), 121-131.

[6] S. S. Chen And L. Greenberg, Hyperbolic spaces, Contributions to analysis, a collection of papers dedicated to Lipman Bers, Academic Press, New York, 1974, 49-87.

[7] S. Friedland AND S. Hersonsky, Jørgensen's inequality for discrete groups in normed algebras, Duke Math. J. 69 (1993), 593-614.

[8] X. Fu, L. Li AND X. WANG, A lower bound for the volumes of complex hyperbolic orbifolds, Geometriae Dedicata 155 (2011), 21-30.

[ 9 ] S. Gallot, D. Hulin and J. Lafontaine, Riemannian geometry, 2nd ed, Springer-Verlag, 1990. 
[10] F. W. Gering and G. J. Martin, Inequalities for Möbius transformations and discrete groups, J. Reine Angew. Math. 418 (1991), 31-76.

[11] W. M. Goldman, Complex hyperbolic geometry, Oxford Math. Monogr., Oxford University Press, 1999.

[12] A. Gray, The volume of a geodesic ball in a Riemanian manifold, Mich. Math. J. 20 (1973), 329-344.

[13] A. Gray, Tubes, Addison-Wesley, Reading, MA, 1990.

[14] S. Hersonsky, Covolume estimates for discrete groups of hyperbolic isometries having parabolic elements, Mich. Math. J. 40 (1993), 467-475.

[15] S. Hersonsky, A generalization of the Shimizu-Leutbecher lemma and Jørgensen inequalities to Möbius transformations in $\mathbf{R}^{n}$, Proc. Amer. Math. Soc. 121 (1994), 209-215.

[16] S. Hersonsky and F. Paulin, On the volumes of complex hyperbolic manifolds, Duke Math. J. 84 (1996), 719-737.

[17] R. A. Horn and C. R. Johnson, Matrix analysis, Cambridge University Press, 1985.

[18] D. A. Kazdan and G. A. Margulis, A proof of Selberg's hypothesis, Math. Sbornik 75 (1968), 162-168.

[19] I. Kim and J. R. Parker, Geometry of quaternionic hyperbolic manifolds, Math. Proc. Camb. Phil. Soc. 135 (2013), 291-320.

[20] G. J. Martin, On discrete Möbius groups in all dimensions: a generalization of Jørensen's inequality, Acta. Math. 163 (1989), 253-289.

[21] G. J. Martin, Balls in hyperbolic manifolds, J. London Math. Soc. 40 (1989), 257-264.

[22] G. D. Mostow, Strong rigidity of locally symmetric space, Princeton University Press, 1973.

[23] J. R. PARKer, Uniform discreteness and Heisenberg translations, Math. Z. 225 (1997), 485-505.

[24] J. R. PARKer, On the volumes of cusped, complex hyperbolic manifolds and orbifolds, Duke Math. J. 94 (1998), 433-464.

[25] W. Thurston, The geometry and topology of 3-manifolds, Lecture Notes, Princeton University, Princeton, 1977.

[26] H. C. WANG, Discrete nilpotent subgroups of Lie groups, J. Differ. Geom. 3 (1969), 481-492.

[27] H. C. WANG, Topics in totally discontinuous groups, in Symmetric spaces, Boothby-Weiss, New York, 1972, 460-485.

[28] P. L. Waterman, An inscribed ball for Kleinian groups, Bull. London Math. Soc. 16 (1984), $525-530$.

[29] N. J. WeilenberG, Discrete Möbius groups: fundamental polyhedra and convergence, Amer. J. Math. 99 (1977), 861-877.

[30] F. ZHANG, Quaternions and matrices of quaternions, Linear Algebra Appl. 251 (1997), $21-57$.

Wensheng Cao

School of Mathematics and Computational Science

WUYI UNIVERSITY

JiANGMEN, GuANGDONG 529020

P.R. CHINA

E-mail: wenscao@aliyun.com 